PoS $\quad \begin{aligned} & \text { PROCEEDINGS } \\ & \text { OF SCIENCE }\end{aligned}$

\title{
Recent soft QCD results from ATLAS and CMS
}

Valentina Maria Martina Cairo*, on behalf of the ATLAS and CMS Collaborations SLAC National Accelerator Laboratory - CA, USA

E-mail: valentina.maria.cairoecern.ch; vcairo@slac.stanford.edu

In this contribution, a review of recent experimental results on soft Quantum Chromodynamics obtained by the ATLAS and CMS Collaborations at the Large Hadron Collider is given.

7th Annual Conference on Large Hadron Collider Physics - LHCP2019

20-25 May, 2019

Puebla, Mexico

${ }^{*}$ Speaker. 


\section{Introduction}

Hard Quantum Chromodynamics (QCD) events constitute only a tiny fraction of the total proton-proton ( $p p)$ cross-section, which is dominated by soft events (peripheral processes): while hard QCD processes can be studied by means of perturbative approaches, this is not possible for the soft QCD events. The development of Monte Carlo (MC) event generators began shortly after the discovery of the partonic structure of hadrons and the formalisation of QCD as the theory of strong interactions: models have to be developed with a set of tunable parameters to describe the hadron-level properties of final states dominated by soft QCD. Thus, soft QCD measurements are crucial for the tuning of the MC event generator and essential to understand and correctly simulate other more complex phenomena. In addition, most of the soft QCD analyses are trackbased measurements, which makes them ideal to study tracking performance in the early stages of a new data-taking period. In Section 2 a recap of charged particle measurements by ATLAS [1] and CMS [2] will be given. In Section 3 the first LHC results on Single Diffractive cross section from both ATLAS plus ALFA [3] and CMS plus TOTEM [4] with an $8 \mathrm{TeV}$ data set will be reported. Section 4 revolves around Underlying Event measurements studied in events with tracks, events with $Z$ bosons or events with top pairs as a leading object, measured by both ATLAS and CMS. Studies of the Underlying Event at forward pseudorapidities with CMS plus CASTOR [5] will also be mentioned. Finally, in Section 5 Double Parton Scattering analyses in the four lepton final state by ATLAS and in same-charge WW events in CMS will be described.

\section{Charged Particle Multiplicity}

Inclusive charged-particle measurements in $p p$ collisions provide insight into the strong interaction in the low energy, non-perturbative QCD region. Charged particles constitute the main source of background when more than one interaction per bunch crossing occurs. Perturbative QCD cannot be used for peripheral interactions: the Non Diffractive (ND) interactions are described by QCD-inspired phenomenological models, while the Single Diffractive (SD) or Double Diffractive (DD) interactions are hardly described and little data are available. Thus, when analysing charged particle distributions the goal is to remove detector effects via an unfolding procedure and measure the spectra of primary charged particles. The ATLAS Experiment has measured unfolded charged particle distributions at $13 \mathrm{TeV}$ in three different phase space regions [6, 7]:

- $\operatorname{track} p_{\mathrm{T}}<500 \mathrm{MeV},|\eta|<2.5[6]$;

- $\operatorname{track} p_{\mathrm{T}}<500 \mathrm{MeV},|\eta|<0.8[6]$;

- $\operatorname{track} p_{\mathrm{T}}<100 \mathrm{MeV},|\eta|<2.5[7]$.

Figure 1 shows the average primary charged-particle multiplicity in $p p$ interactions per unit of pseudorapidity $\eta$ for $|\eta|<0.2$ as a function of the centre-of-mass energy $\sqrt{s}$. The predictions from EPOS [8] and Pythia 8 with the MONASH tune [9] match the data well. For Pythia 8 with the A2 tune [10], the match is not as good as was observed when measuring particles with $p_{\mathrm{T}}<$ $500 \mathrm{MeV}$ [6]. 


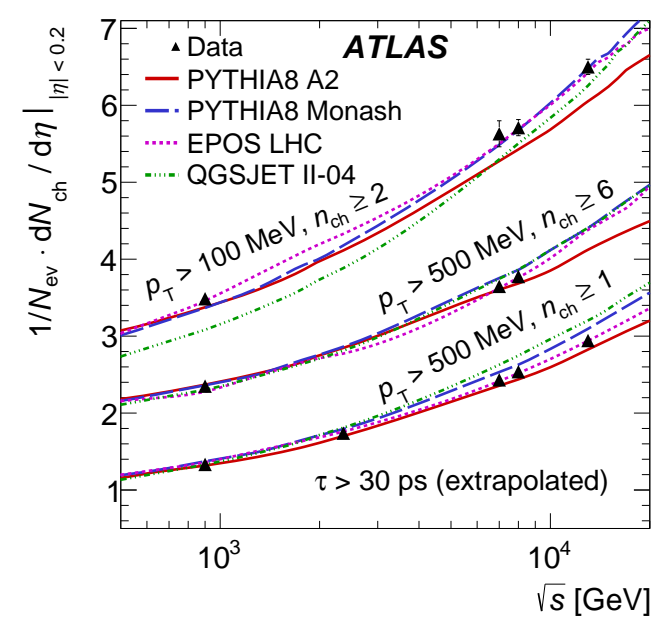

Figure 1: The average primary charged-particle multiplicity in $p p$ interactions per unit of pseudorapidity $\eta$ for $|\eta|<0.2$ as a function of the centre-of-mass energy $\sqrt{s}$. The data are shown as black triangles with vertical errors bars representing the total uncertainty. They are compared to various MC predictions which are shown as coloured lines. Ref. [7].

The CMS experiment has measured charged particle multiplicity in heavy ion collisions too, for instance in 5.44 TeV Xe-Xe collisions [11], as shown in Figure 2 for various intervals of centrality, which is related to the impact parameter of the collision and represents a percentile of the total nuclear interaction cross section, e.g. the most central collisions are denoted by the lowest percentiles.

EPOS LHC [8] gives the best predictions of charged particle multiplicities both in proton and heavy ion collisions.

Some large discrepancies between data and MC predictions remain, in particular in the low charged particle multiplicity regime, as shown in Figures 3(a) and 3(b). This region is dominated by diffractive events, as can be seen in Figure 3(c), thus measurements of the diffractive cross section in $p p$ collisions are fundamental to tune the $\mathrm{MC}$ event generators in this regime.

\section{Single Diffractive Cross-Section}

Single diffractive dissociation constitutes $10 \%$ of the total $p p$ cross-section: it takes place via the exchange of a net colour-singlet strongly interacting object, a Pomeron. The applicability of a universal Pomeron across total, elastic and diffractive processes has a long history of investigation. Diffraction is also an important ingredient in understanding the low Bjorken- $x$ region of proton structure and cosmic ray air showers, and it may even be related to the string theory of gravity [12]. Experimentally, diffractive events can be selected mainly in two ways:

- by exploiting "large rapidity gaps" - with this approach it is not possible to isolate SD from $\mathrm{DD}$ and ND and to access the squared 4-momentum transfer $t$ and the energy loss $\xi$ of the proton; 

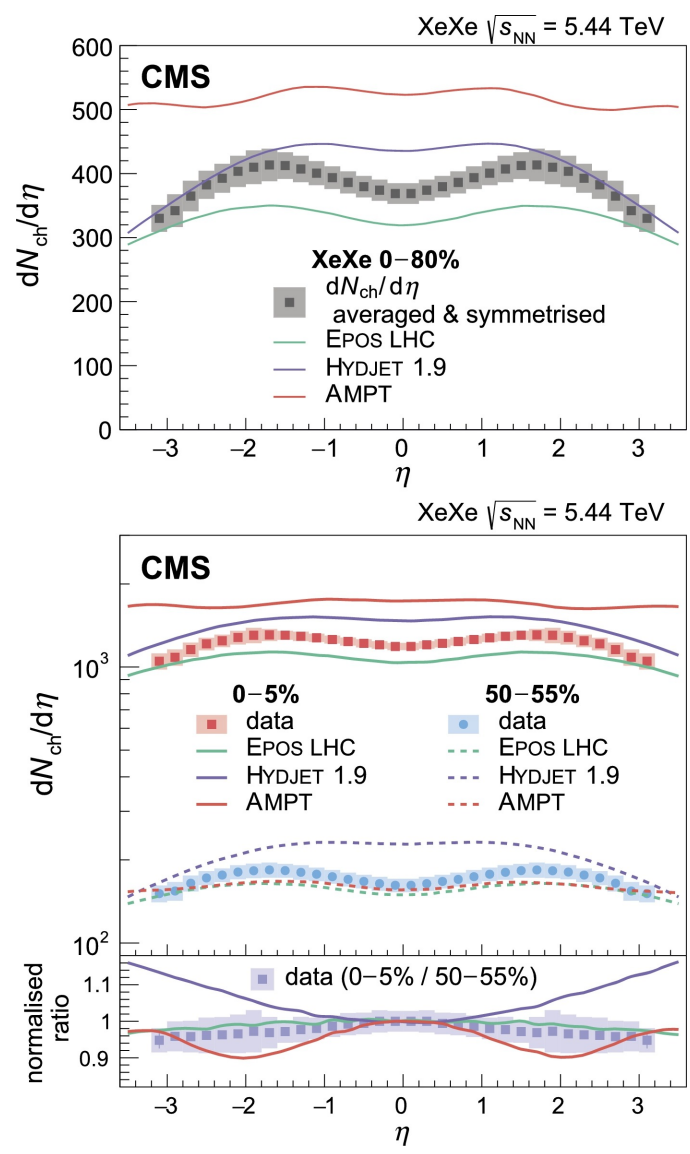

Figure 2: Averaged and symmetrised charged-particle multiplicity per unit of pseudorapidity $d N_{\mathrm{ch}} / d \eta$ in $\mathrm{XeXe}$ collisions at $\sqrt{s_{N N}} 5.44 \mathrm{TeV}$ (grey squares), for events in the 0-80\% centrality interval (upper), as well as the $0-5 \%$ (red squares) and 50-55\% (blue circles) centrality intervals (lower). Predictions from various event generators are also shown for comparison. The ratios of the $d N_{\mathrm{ch}} / d \eta$ distributions for events in the $0-5 \%$ to those in the $50-55 \%$ centrality interval, normalised to unity at midrapidity, are shown in the bottom panel. The bands around the data points denote the total systematic uncertainties, while the statistical uncertainties are negligible. Ref. [11].

- by detecting the scattered proton - with this approach, a direct access to $t$ is possible and the other diffractive contributions are suppressed.

Both ATLAS and CMS have measured diffractive cross-sections through large rapidity gaps in the past $[13,14]$ and have recently released the first SD differential cross-section measurements through direct detection of the intact proton in the forward detectors ALFA [3] and TOTEM [4]. These measurements have been carried out with an $8 \mathrm{TeV}$ dedicated dataset collected in July 2012 with special LHC beam configurations leading to a number of contemporary interactions per crossing $\mu<0.08$ and $\beta^{*}=90 \mathrm{~m}$.

Figure 4 shows the hadron level cross-section measured by ATLAS and ALFA as a function of the rapidity gaps $\Delta \eta$. For gap sizes between about 1.5 and 3.5, the differential cross section exhibits the plateau that is characteristic of rapidity gap distributions in soft diffractive processes. There are deviations from this behaviour at smaller and larger gap sizes due to the definition of the observable 


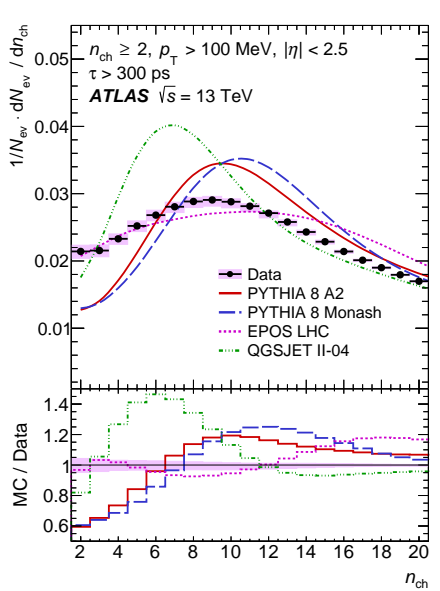

(a)

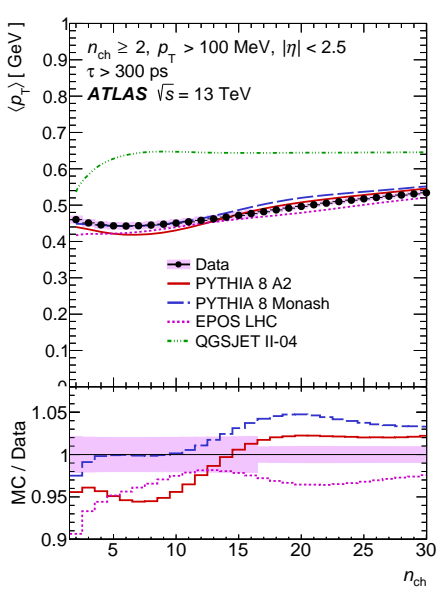

(b)

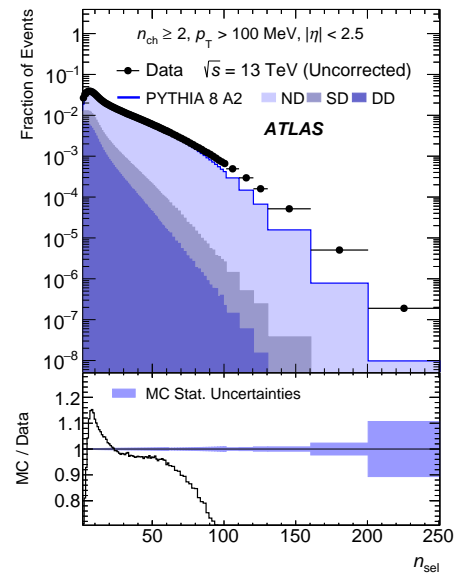

(c)

Figure 3: Figures 3(a) and 3(b) show, respectively, the number of charged particles $N_{\text {ch }}$ and the mean transverse momentum $\left\langle p_{\mathrm{T}}\right\rangle$ versus the number of charged particles $N_{\mathrm{ch}}$ in the low multiplicity region. Figure 3(c) shows the comparison of the uncorrected data and Pythia 8 A2 simulation for the number of selected reconstructed tracks per event. The same selection is applied to data and MC. All the distributions represent events with at least two primary charged particles with $p_{\mathrm{T}}>100 \mathrm{MeV}$ and $|\eta|<2.5$. Ref. [7].

in terms of a restricted rapidity region corresponding to the Inner Detector acceptance, and to the fiducial range restriction, respectively. The data are compared with the SD process simulations in the A2 [10] and A3 [15] tunes of Pythia 8, which exceed the measurement by factors of 2.3 and 1.5, respectively. Both of these tunes are based on an integrated SD cross section, defined according to the Pythia 8 model, of $12.5 \mathrm{mb}$. The difference between their predictions for the fiducial region of the measurement arises from the different Pomeron intercepts $\alpha(0)$ in their flux factors. Both models give a reasonable description of the shape of the $\Delta \eta$ distribution, the $\mathrm{A} 2$ tune being slightly better than A3. The excess of the Pythia 8 prediction over data is compatible with previous ATLAS observations from rapidity gap spectra assuming the DD contribution to the Pythia 8 model of the previous measurement is correct. The Herwig $7[16,17]$ prediction is also broadly in line with the shape of the $\Delta \eta$ distribution, but exhibits an even larger excess in normalisation. This may be partly due to the operational definition of the SD process that is adopted in the default SD model normalisation, which is derived from a rapidity gap measurement that also contains a DD admixture [18].

In both ATLAS and CMS analyses, the differential diffractive cross section is also presented as a function of the squared four-momentum transfer $t$ in the $p p$ collision, as shown in 5(a) and 5(b) respectively. The CMS measurement exhibits slightly larger uncertainties, but it covers a larger range in $|t|$. The current data do not show yet conclusive evidence for a flattening of the distribution in larger $|t|$ region as it was observed by CDF [19]. A more extensive discussion can be found in [4] and references therein.

\section{Underlying Event}

The Underlying Event (UE) can be defined as the activity accompanying any hard scattering 


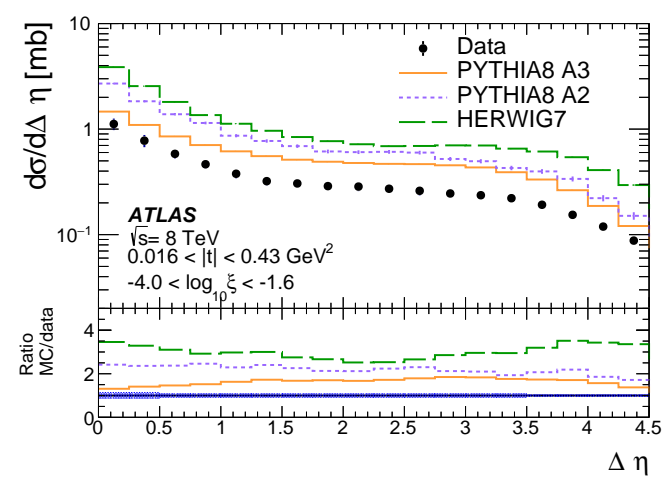

Figure 4: Measured hadron level differential cross section as a function of $\Delta \eta$, comparing with MC predictions. The error bars display the combination of statistical and systematic uncertainties in quadrature. Ref. [3].

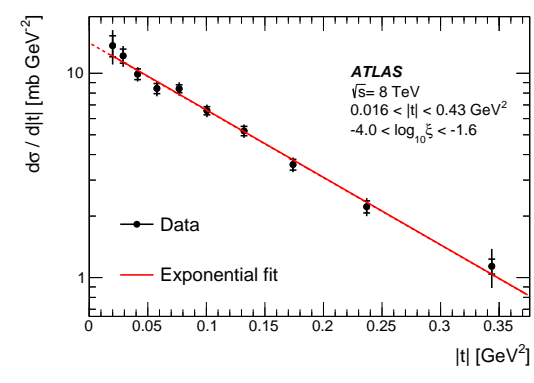

(a)

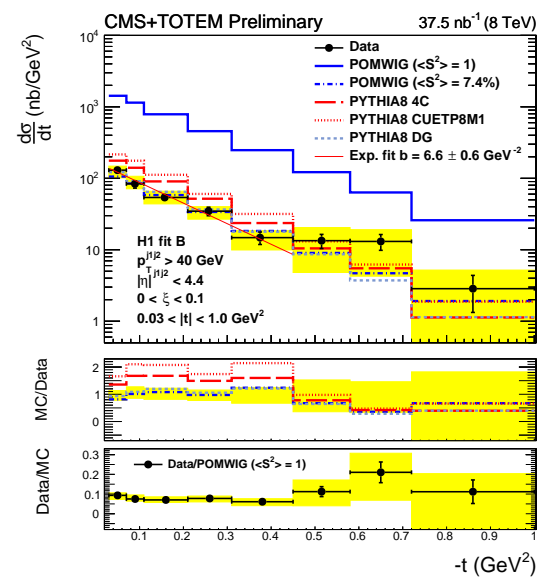

(b)

Figure 5: The differential cross section as a function of $|t|$ for 5(a) ATLAS and 5(b) CMS respectively. Ref. $[3,4]$.

in a collision event and it can arise from:

- Partons not participating in a hard-scattering process (beam remnants);

- Multiple parton interactions (MPI);

- Initial and final state gluon radiation (ISR, FSR).

Measurements of the UE associated with different hard processes (leading track, jet, $Z$ boson, top pair) are useful to test the level of universality of the underlying MPI dynamics. Events with a harder scale are expected to correspond, on average, to proton-proton interactions with a smaller impact parameter and therefore with more MPI. Such increased UE activity is observed to plateau at high energy scales, which indicates that the smallest impact parameters have been reached and hence maximum matter overlap in the $p p$ collision. Observables can be defined which are sensitive 
to the UE. For instance the average number of charged particles per event measured as a function of the angle in the transverse plane of the colliding beams with respect to an object with high transverse momentum ("leading object"), as schematically shown in Figure 6.

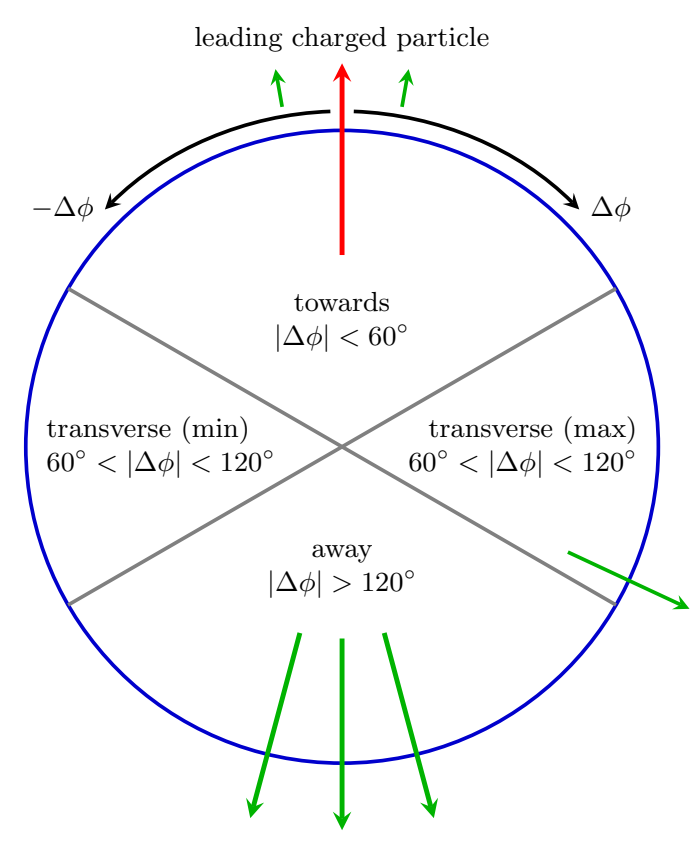

Figure 6: Definition of regions in the azimuthal angle with respect to the leading (highest- $p_{\mathrm{T}}$ ) charged particle, with arrows representing particles associated with the hard scattering process and the leading charged particle highlighted in red. Conceptually, the presence of a hard-scatter particle on the right-hand side of the transverse region, increasing its $\sum p_{\mathrm{T}}$, typically leads to that side being identified as the trans-max and hence the left-hand side as the trans-min, with maximum sensitivity to the UE. Ref. [20].

The ATLAS Collaboration has performed a track based underlying event analysis in $13 \mathrm{TeV}$ data [20], which follows closely the charged particle selection used in [6] mentioned in Section 2.

Figure 7 shows the mean density of charged-particle multiplicity $N_{\text {ch }}$ as a function of the azimuthal angle $\Delta \phi$ (with respect to the leading charged particle). Two event selections are shown: the $p_{\mathrm{T}}^{\text {lead }}>1 \mathrm{GeV}$ cut common to all observables, and a harder $p_{\mathrm{T}}^{\text {lead }}>10 \mathrm{GeV}$ requirement. The difference between these two selections illustrates the transition from relatively isotropic minimumbias scattering to the emergence of hard partonic scattering structure and hence a dominant axis of energy flow. This event structure with least activity perpendicular to the leading-object axis, i.e. away from $\Delta \phi=0$ or $180^{\circ}$, is seen for both selections and both observables but is much stronger for the event subset with the higher $p_{\mathrm{T}}^{\text {lead }}>10 \mathrm{GeV}$ requirement: this demonstrates the evolution of event shape as a hard scattering component develops. There is no clear "best" MC model for these observables. Consistent with the observations of Section 2, EPOS performs best in the more inclusive $p_{\mathrm{T}}^{\text {lead }}>1 \mathrm{GeV}$ selection.

In addition, both ATLAS and CMS have measured observables sensitive to the Underlying Event in $Z \rightarrow \mu \mu$ events [21, 22].

Figure 8 shows the distributions of $N_{\mathrm{ch}}$ measured at $\sqrt{s}=13 \mathrm{TeV}$ compared with the results of previous ATLAS and CDF measurements [21]. The relative uncertainties of the two ATLAS measurements are of similar sizes, while the CDF measurements have large statistical fluctuations. 


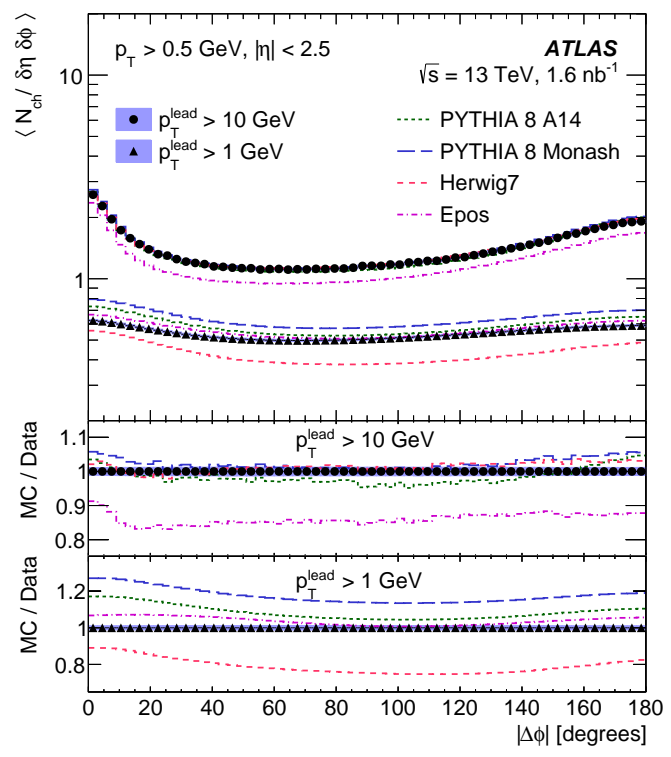

Figure 7: Distribution of mean density of charged-particle multiplicity $N_{\mathrm{ch}}$ as a function of $\Delta \phi$ (with respect to the leading charged particle) for $p_{\mathrm{T}}^{\text {lead }}>1 \mathrm{GeV}$ and $p_{\mathrm{T}}^{\text {lead }}>10 \mathrm{GeV}$ separately, with comparisons to MC generator models. The error bars on data points represent statistical uncertainty and the blue band the total combined statistical and systematic uncertainty. Ref. [20].

All three measurements reveal the turn-on effect of the UE activity in the fiducial region. With higher centre-of-mass energies, more energy is available for the processes forming the UE e.g. MPI. Hence, the rise of the UE activity as a function of $\sqrt{s}$ is expected.

Figure 9 shows the UE activity, with a $p_{\mu \mu}<5 \mathrm{GeV}$ requirement, as a function of $\sqrt{s}$ for data compared to model predictions. There is a significant increase, by a factor 2-2.5, as the collision energy rises from 1.96 to $13 \mathrm{TeV}$, which is qualitatively reproduced by Powheg [23]. The energy evolution is better described by Powheg with Pythia 8, whereas hadronization with Herwig++ overestimates the UE activity at all collision energies. The comparison of the distributions with and without MPI indicates that the ISR and FSR contributions, which increase slowly with centerof-mass energy, are small. The CUETP8M1 [24] tune of Pythia 8 and the EU-EE-5C tune of Herwig++ [25] employed here are mostly obtained from fits to minimum-bias measurements and UE measurements with leading jets or leading tracks. The fact that these tunes reproduce globally well the present data supports the hypothesis that the UE activity is independent of the hard process. The present study also confirms that the collision energy dependence of the UE activity is similar for different hard processes.

Unlike UE studies with a leading track/jet, these measurements provide new handles to better understand the evolution of ISR, FSR, and MPI contributions separately, as functions of the event energy scale and the collision energy.

The CMS Collaboration has also characterized the Underlying Event in top-quark pairs [26]. The study of the UE in top pair events provides a direct test of its universality at higher energy scales than those probed in minimum bias or Drell-Yan events. This is relevant as a direct probe of color reconnection (CR), which is needed to confine the initial QCD color charge of the top quark 


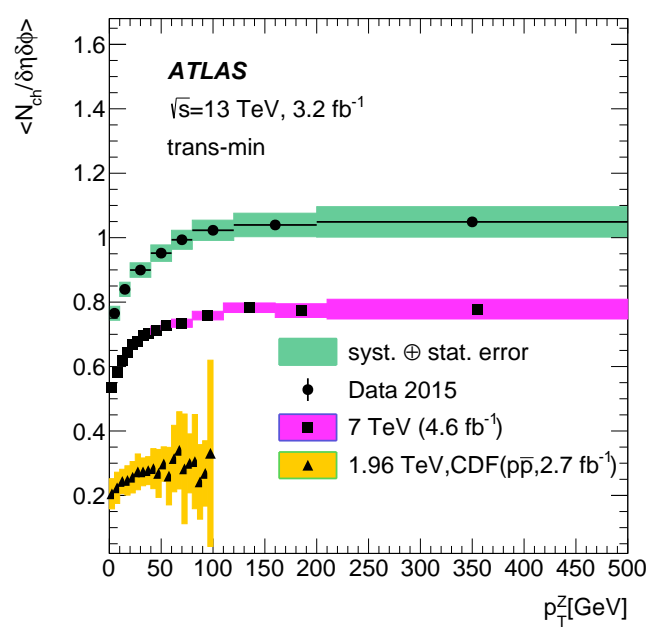

Figure 8: The distributions of $N_{\mathrm{ch}}$ measured at $\sqrt{s}=13 \mathrm{TeV}$ compared with the results of the previous ATLAS and CDF measurements. The error bars correspond to the full uncertainties of the corresponding measurement. Ref. [21].

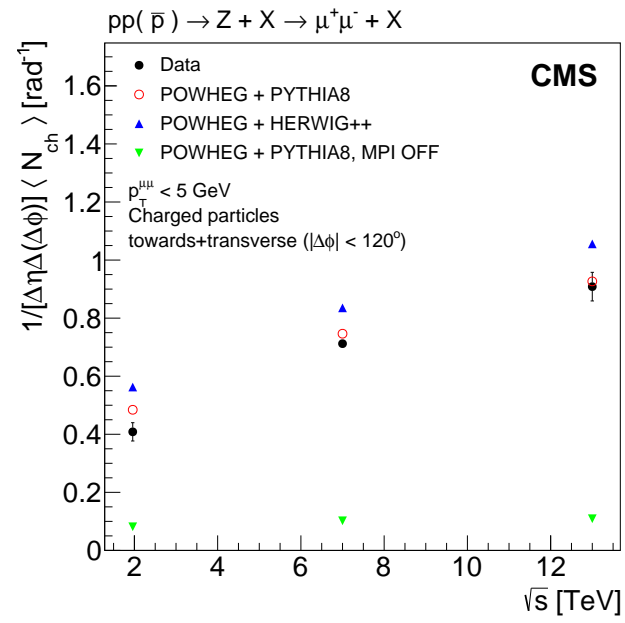

Figure 9: Average particle density for $\mathrm{Z}$ events as a function of the center-of-mass energy, measured in the combined towards plus transverse regions, compared to MC predictions. The error bars represent the statistical and systematic uncertainties added in quadrature. Ref. [22].

into color-neutral states. The CR mainly occurs between one of the products of the fragmentation of the $b$ quark from the top quark decay and the proton remnants. This is expected to induce an ambiguity in the origin of some of the final states present in a bottom quark jet. The impact of these ambiguities in the measurement of top quark properties is evaluated through phenomenological models that need to be tuned to the data. Recent examples of the impact that different model parameters have on the top mass can be found in Refs. [27, 28].

For instance, Figure 10 represents the average sphericity $\mathrm{S}$, which measures the $p_{\mathrm{T}}^{2}$ with respect to the axis of the event. An isotropic (dijet) event is expected to have $S=1(0)$. The average sphericity increases significantly when no extra jets are present in the event showing that the UE 
is slightly more isotropic in these events. The MPI contribution is crucial: most of the pulls are observed to be very large when MPI is switched off in the simulation. Color reconnection effects are, on the other hand, more subtle and are more relevant when no additional jet is present in the event. This is illustrated by the fact that the pulls of the setup without CR are larger for events belonging to these categories.
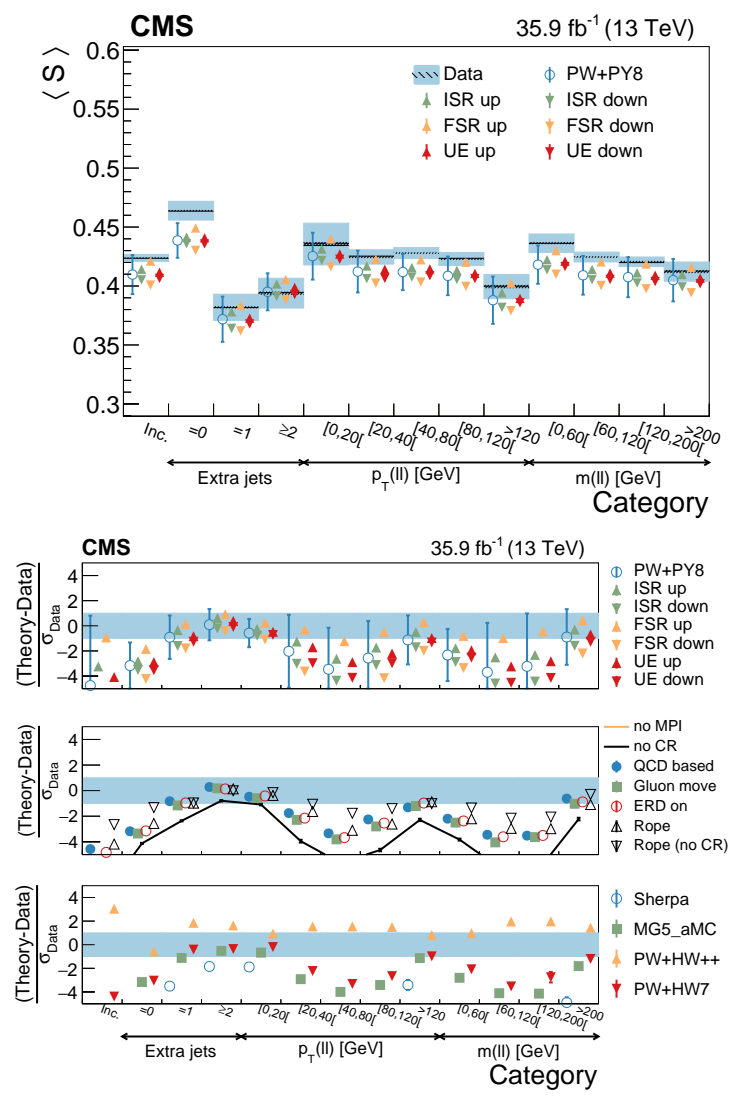

Figure 10: Average sphericity in different categories. The mean observed in data (boxes) is compared to the predictions from different models (markers), which are superimposed in the upper figure. The total (statistical) uncertainty of the data is represented by a shaded (hatched) area and the statistical uncertainty of the models is represented with error bars. In the specific case of the POWHEG+Pythia 8 model the error bars represent the total uncertainty. The lower figure displays the pull between different models and the data, with the different panels corresponding to different sets of models. The bands represent the interval where Ipull|<1. The error bar for the POWHEG+Pythia 8 model represents the range of variation of the pull for the different configurations described in the text. Ref. [26].

Furthermore, the energy carried by particles produced in the very forward region covered by the CASTOR calorimeter [5] of the CMS experiment is a powerful probe of the activity of the Underlying Event. The measurements presented in Ref. [29] provide the first correlation of hadron activity at very forward and central rapidities performed at a centre-of-mass energy of $13 \mathrm{TeV}$ providing a new approach to the study of hadron production at the LHC. Such measurements, spanning huge rapidity intervals, provide additional information on the underlying event compared to measurements using only information from the central region. The data suggest a nontrivial correspondence between the activity in the very forward and the central regions. The very forward 
region covered by the data contains the highest energy densities studied in proton-proton collisions at the LHC. The present results can therefore be also used to improve the predictions of event generators used in studies of cosmic rays that induce extensive air showers at ultra-high energies [30].

The results in Ref. [29] show that the average total energy in CASTOR increases with the track multiplicity. This feature is consistent with the general behaviour of the underlying event measured at central rapidities as discussed above and is reproduced by all models considered. The systematic uncertainty of the data, though, is dominated by the energy scale uncertainty contribution, which is fully correlated between the multiplicity bins. Therefore, the distributions can be normalised to the first bin, so that, when comparing their shapes, the systematic uncertainty is significantly smaller. Figure 11 shows that the relative increase is steep at low multiplicities and becomes softer at higher multiplicities. All Pythia 8 tunes have very similar shapes, inconsistent with that observed in the data. All the other generators see a saturation at about $N_{\mathrm{ch}}>80$, not visible in data. The worst predictions are given by EPOS LHC, which instead showed good prediction of the energy flow in the calorimeter in minimum bias-like events in previous ATLAS measurements [31]. The data therefore provide relevant information for future generator improvements and tunes.

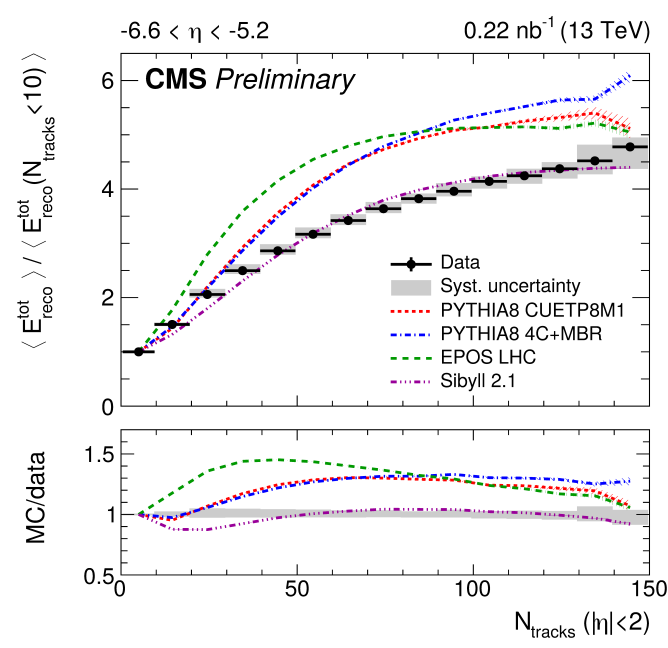

Figure 11: Average total energy reconstructed in the CASTOR calorimeter normalised to that in the first bin $\left(N_{\mathrm{ch}}<10\right)$ as a function of the number of reconstructed tracks for $\Delta \eta<2$. In all figures, the data are shown as black circles and the corresponding systematic uncertainties with a gray band; horizontal bars are used to indicate the bin width. The predictions of various event generators are compared to the data, which are the same in both panels. The bands associated with the model predictions illustrate the model uncertainty. Ref. [29].

\section{Double Parton Scattering}

One source of the underlying-event activity is MPI. In high-energy $p p$ interactions, where the density of low- $x$ partons is high, there is enough energy to produce hard multi-parton interactions. The simplest example is hard double-parton scattering (DPS): two partons from each proton interact with each other leading to perturbative final states. The interest in studying DPS is twofold: 
- the probability of it and the potential correlations between the products of these two perturbative interactions provide valuable information about the dynamics of the partonic structure of the proton;

- DPS processes may also constitute a background to reactions proceeding through singleparton scattering (SPS).

Both the ATLAS and CMS collaborations have released DPS measurements. ATLAS has investigated DPS in four-lepton final states [32], which constitute the decay products of two $Z$ bosons produced in two distinct parton-parton scatterings within the same $p p$ interaction. Since double Drell-Yan production is driven by quark-antiquark annihilation, while most of the previously explored DPS processes are driven by gluon-gluon scattering, and the final state of four charged leptons constitutes the golden channel for the studies of Higgs boson properties, $H \rightarrow Z Z \rightarrow 4 l$, a study of a possible DPS contribution to the production of four isolated charged leptons at $8 \mathrm{TeV}$ is warranted. No signal of double-parton scattering was observed and an upper limit on the fraction of the DPS contribution to the inclusive four-lepton final state of 0.042 was obtained at 95\% CL. This translates into a lower limit of $1.0 \mathrm{mb}$ on the effective cross section.

One of the most promising processes to study DPS is the case in which both hard scatterings lead to the production of a $W$ boson, and in particular the final state with two same-charge $W$ bosons.

$W W$ production from a single hard scatter (SHS) includes two additional partons carrying high transverse momenta and is thus suppressed at the matrix-element level. The absence of jets in the same-charge $W$ boson pair production via DPS at LO in perturbation theory provides an extra handle to reduce contributions from the SHS backgrounds by introducing an upper requirement on the number of jets. Moreover, if both $W$ bosons decay leptonically, this process exhibits a clean final state in the detector, and the excellent reconstruction and resolution of leptons in the CMS detector allow for an accurate study of its properties. To date, this process has not yet been observed experimentally. An eventual observation of this process would permit to study the validity of the factorization approach, which is prevalent in current MC event generators. The DPS WW process constitutes also a background in searches for new physics at the LHC and the determination of its production cross section would improve the reach of such searches.

The CMS collaboration has recently found [33] in $13 \mathrm{TeV} p p$ data the first evidence for WW production via DPS, with a significance of 3.9 standard deviations. The measured inclusive cross section, shown in Figure 12 is $1.41 \pm 0.28$ (stat) \pm 0.28 (syst) pb.

\section{Conclusions}

The LHC and its experiments allow for extensive tests of soft QCD. The predictions from MC event generators have very much improved since Run 1, but there are still visible discrepancies when compared to data collected by ATLAS and CMS, in particular for the underlying event at larger momentum transfer. A good modelling of soft QCD is crucial for many more complex analyses, e.g. precision measurements of the top quark mass [34]. While the existing soft measurements already challenge the models, there are plenty of possibilities to guide theory with new measurements. 


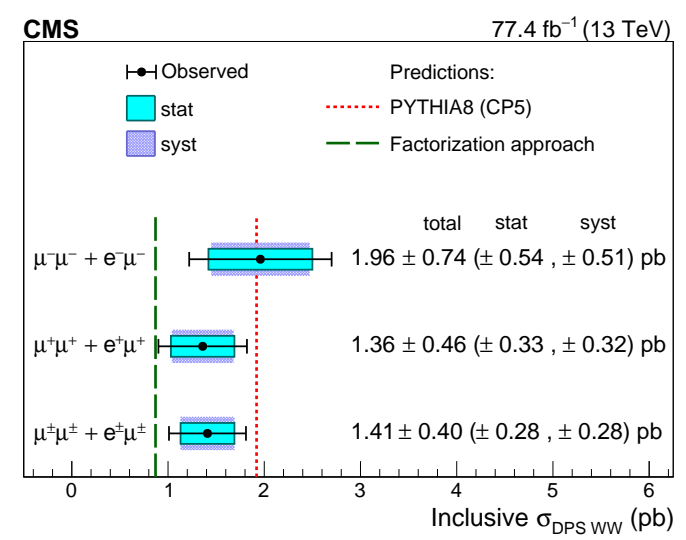

Figure 12: Observed cross section values for inclusive DPS WW production from the two lepton charge configurations and their combination. These values are obtained from the extrapolation of the observed DPS WW cross section to the inclusive WW case. The statistical and systematic uncertainties are shown using shaded bands. The predictions from Pythia and from the factorization approach are represented with the red dotted and green dashed lines, respectively. Ref. [33].

\section{References}

[1] ATLAS Collaboration. "The ATLAS Experiment at the CERN Large Hadron Collider". In: JINST 3 (2008), S08003. DOI: 10.1088/1748-0221/3/08/S08003.

[2] CMS Collaboration. “The CMS experiment at the CERN LHC". In: JINST 3 (2008), S08004. DOI: $10.1088 / 1748-0221 / 3 / 08 / 508004$.

[3] ATLAS Collaboration. "Measurement of Differential Cross Sections for Single Diffractive Dissociation in $\sqrt{s}=8 \mathrm{TeV} p p$ collisions using the ATLAS ALFA Spectrometer". ATLASCONF-2019-012. 2019. URL: https: / / cds . cern. ch/record/2670978.

[4] CMS and Totem Collaborations. "Measurement of dijet production with a leading proton in proton-proton collisions at $\sqrt{s}=8 \mathrm{TeV} p p^{\prime}$. CMS-PAS-FSQ-12-033, CERN-TOTEMNOTE-2018-001. 2018. uRL: https : / cds . cern. ch/record/2638198.

[5] V. et al Andreev. "Performance studies of a full-length prototype for the CASTOR forward calorimeter at the CMS experiment". In: Eur. Phys. J. C 67.3 (2010), pp. 601-615. ISSN: 1434-6052. DOI: 10 . 1140 / epjc/s10052-010-1316-4. URL: https : / / doi . org/10.1140/epjc/s10052-010-1316-4.

[6] ATLAS Collaboration. "Charged-particle distributions in $\sqrt{s}=13 \mathrm{TeV} p p$ interactions measured with the ATLAS detector at the LHC". In: Phys. Lett. B 758 (2016), p. 67. DOI: $10.1016 / j$.physletb.2016.04.050. arXiv: 1602.01633 [hep-ex] .

[7] ATLAS Collaboration. "Charged-particle distributions at low transverse momentum in $\sqrt{s}=$ $13 \mathrm{TeV} p p$ interactions measured with the ATLAS detector at the LHC'. In: Eur. Phys. J. C 502 (2016), p. 76. DOI: 10.1140 /epjc/s10052-016-4335-y. arXiv: 1606.01133 [hep-ex]. 
[8] S. Porteboeuf, T. Pierog, and K. Werner. "Producing Hard Processes Regarding the Complete Event: The EPOS Event Generator". In: (2010). arXiv: 1006.2967 [hep-ph] .

[9] Peter Skands, Stefano Carrazza, and Juan Rojo. "Tuning PYTHIA 8.1: the Monash 2013 Tune". In: Eur. Phys. J. C74.8 (2014), p. 3024.

[10] The ATLAS Collaboration. "Further ATLAS tunes of PYTHIA6 and Pythia 8". eprint: ATLPHYS-PUB-2011-014.

[11] CMS Collaboration. "Pseudorapidity distributions of charged hadrons in xenon-xenon collisions at $\sqrt{s}=5.44 ; \mathrm{TeV} p p$ ”. In: Phys. Lett. B 799 (2019). arXiv: 1902.03603 [hep-ex ] .

[12] Richard C Brower et al. "The Pomeron and gauge/string duality". In: JHEP 2007.12 (2007), 005-005. ISSN: 1029-8479. DOI: 10.1088/1126-6708/2007/12/005. URL: http: //dx.doi.org/10.1088/1126-6708/2007/12/005.

[13] ATLAS Collaboration. "Dijet production in $\sqrt{s}=7 \mathrm{TeV} p p$ collisions with large rapidity gaps at the ATLAS experiment”. In: Phys. Lett. B 754 (2016), p. 214. DOI: $10.1016 / j$. physletb.2016.01.028. arXiv: 1511.00502 [hep-ex].

[14] CMS Collaboration. "Study of dijet events with a large rapidity gap between the two leading jets in $p p$ collisions at $\sqrt{s}=7$ TeV". In: Eur. Phys. J. C 78 (2018), p. 242. DOI: $10.1140 /$ epjc/s10052-018-5691-6. arXiv: 1710.02586 [hep-ex] .

[15] ATLAS Collaboration. "The Pythia 8 A3 tune description of ATLAS minimum bias and inelastic measurements incorporating the Donnachie-Landshoff diffractive model". ATLPHYS-PUB-2016-017. 2016. URL: https : / cds . cern.ch/record/2206965.

[16] M. Bahr et al. "Herwig++ Physics and Manual”. In: Eur. Phys. J. C58 (2008), pp. 639-707.

[17] J. Bellm et al. "Herwig 7.0/Herwig++ 3.0 release note". In: Eur.Phys.J. C76 (2016), p. 196.

[18] S. Gieseke, F. Loshaj, and P. Kirchgaeber. "Soft and diffractive scattering with the cluster model in Herwig". In: Eur. Phys. J. C77 (2017). arXiv: 1612 . 04701.

[19] CDF Collaboration. "Diffractive dijet production in $p \bar{p}$ collisions at $\sqrt{s}=1.96 \mathrm{TeV}$ ". In: Phys. Rev. D. 86 (2012). DOI: 10 . 1103 / PhysRevLett . 88 . 151802, arXiv: $0109025 \mathrm{v} 1$.

[20] ATLAS Collaboration. "Measurement of charged-particle distributions sensitive to the underlying event in $\sqrt{s}=13 \mathrm{TeV}$ proton-proton collisions with the ATLAS detector at the LHC”. In: JHEP 03 (2017), p. 157. DOI: 10.1007 / JHEP 03 (2017) 157. arXiv: 1701. 05390 [hep-ex].

[21] ATLAS Collaboration. "Measurement of distributions sensitive to the underlying event in inclusive Z-boson production in $p p$ collisions at $\sqrt{s}=13 \mathrm{TeV}$ ) with the ATLAS detector". 2019. arXiv: 1905.09752 [hep-ex].

[22] CMS Collaboration. "Measurement of the underlying event activity in inclusive $Z$ boson production in proton-proton collisions at $\sqrt{s}=13 \mathrm{TeV}$ ". In: JHEP 07 (2018), p. 032. DOI: 10.1007/JHEP 07 (2018) 032. arXiv: 1711.04299 [hep-ex]. 
[23] Stefano Frixione, Paolo Nason, and Carlo Oleari. "Matching NLO QCD computations with parton shower simulations: the POWHEG method". In: JHEP 2007.11 (2007), pp. 070-070. DOI: $10.1088 / 1126-6708 / 2007 / 11 / 070$. URL: https://doi.org/10.1088\% $2 \mathrm{~F} 1126-6708 \div 2 \mathrm{~F} 2007 \div 2 \mathrm{~F} 11 \div 2 \mathrm{~F} 070$.

[24] CMS Collaboration. "Event generator tunes obtained from underlying event and multiparton scattering measurements". In: Eur. Phys. J. C 76.3 (2016), p. 155. ISSN: 1434-6052. DOI: $10.1140 /$ epjc/s10052-016-3988-x. URL: https://doi.org/10.1140/ epjc/s10052-016-3988-x.

[25] M. H. Seymour and A. Siódmok. "Constraining MPI models using $\sigma$ eff and recent Tevatron and LHC Underlying Event data". In: JHEP 2013.10 (2013), p. 113. ISSN: 1029-8479. DOI: 10 . 1007 / JHEP10 (2013) 113. URL: https : / / doi . org/10 . 1007/ JHEP10 (2013) 113.

[26] CMS Collaboration. "Study of the underlying event in top quark pair production in $p p$ collisions at $\sqrt{s}=13$ TeV”. In: Eur. Phys. J. C 79 (2019), p. 123. DOI: 10.1140 / epjc/ s10052-019-6620-z. arXiv: 1807.02810 [hep-ex].

[27] Gennaro Corcella, Roberto Franceschini, and Doojin Kim. "Fragmentation uncertainties in hadronic observables for top-quark mass measurements". In: Nucl. Phys. B 929 (2018), pp. 485 -526. ISSN: 0550-3213. DOI: https : / doi.org/10.1016/j.nuclphysb. 2018.02.012. URL: http://www.sciencedirect.com/science/article/ pii/s0550321318300579.

[28] Silvia Ferrario Ravasio et al. "A theoretical study of top-mass measurements at the LHC using NLO+PS generators of increasing accuracy”. In: Eur. Phys. J. C 78.6 (2018), p. 458. ISSN: 1434-6052. DOI: $10.1140 /$ epjc/s10052-018-5909-7. URL: https : //doi.org/10.1140/epjc/s10052-018-5909-7.

[29] CMS Collaboration. "Measurement of the average very forward energy as a function of the track multiplicity at central rapidities in proton-proton collisions at $\sqrt{s}=13 \mathrm{TeV}$ ". CMS PAS FSQ-18-001. 2018. URL: https://cds . cern. ch/record/2669256.

[30] David d'Enterria et al. "Constraints from the first LHC data on hadronic event generators for ultra-high energy cosmic-ray physics”. In: Astroparticle Physics 35.2 (2011), pp. 98 -113. ISSN: 0927-6505. DOI: https://doi .org/10.1016/j.astropartphys. 2011. 05.002. URL: http://www.sciencedirect.com/science/article/pii/ S0927650511000867.

[31] ATLAS Collaboration. "Measurements of the pseudorapidity dependence of the total transverse energy in proton-proton collisions at $\sqrt{s}=7 \mathrm{TeV} p p$ with ATLAS". In: JHEP 11 (2012), p. 033. DOI: 10.1007 / JHEP11 (2012) 033. arXiv: 1208.6256 [hep-ex] .

[32] ATLAS Collaboration. "Study of the hard double-parton scattering contribution to inclusive four-lepton production in $p p$ collisions at $\sqrt{s}=8 \mathrm{TeV}$ with the ATLAS detector". In: Phys. Lett. B 790 (2019), p. 595. DOI: $10.1016 /$ j . physletb. 2019.01 . 062. arXiv: 1811.11094 [hep-ex]. 
[33] CMS Collaboration. "Evidence for WW production from double-parton interactions in protonproton collisions at $\sqrt{s}=13 \mathrm{TeV}$ ". 2019. arXiv: 1909.06265 [hep-ex] .

[34] CMS Collaboration. "Measurement of the top quark mass with lepton+jets final states using $p p$ collisions at $\sqrt{s}=13$ TeV". In: Eur. Phys. J. C 78 (2018), p. 891. DOI: $10.1140 /$ epjc/s10052-018-6332-9. arXiv: 1805.01428 [hep-ex]. 